\title{
Influence of laser wavelength and pulse duration on the degradation of polymeric films embedding photochromic molecules
}

\author{
Athanassia Athanassiou $^{\mathrm{a}, *}$, Despina Sahinidou ${ }^{\mathrm{a}, \mathrm{b}}$, Valentina Arima ${ }^{\mathrm{c}}$, Savas Georgiou $^{\mathrm{a}}$, \\ Roberto Cingolani ${ }^{\mathrm{c}}$, Costas Fotakis ${ }^{\mathrm{a}, \mathrm{b}}$ \\ ${ }^{a}$ Institute of Electronic Structure and Laser (IESL), Foundation for Research and Technology-Hellas (FORTH), 711 10 Heraklion, Crete, Greece \\ ${ }^{\mathrm{b}}$ University of Crete, Physics Department, 71003 Heraklion, Crete, Greece \\ ${ }^{\mathrm{c}}$ National Nanotechnology Laboratory, INFM, CNR, Via Arnesano, 73100 Lecce, Italy
}

Received 1 December 2005; received in revised form 24 February 2006; accepted 13 March 2006

Available online 28 March 2006

\begin{abstract}
An extensive investigation of the photodegradation of the photochromic molecule $1^{\prime}, 3^{\prime}$-dihydro-1', $3^{\prime}, 3^{\prime}$-trimethyl-6-nitrospiro[2 $H$-1-benzopyran$2,2^{\prime}-(2 H)$-indole] in a polymer matrix is studied under irradiation with ultraviolet (UV) laser pulses of 248 and $308 \mathrm{~nm}$ wavelength. Apart from the effect of the wavelength, the effect of the pulse duration is also presented using pulses of $30 \mathrm{~ns}$ and $500 \mathrm{fs}$ for the $248 \mathrm{~nm}$ wavelength. To tackle the problem of oxidative degradation upon irradiation of the photochromic molecules which is known to play a principal role in the photodegradation process, we incorporate Iron(II) phthalocyanine molecules in the examined matrices. These molecules upon UV irradiation are dissociated into photoproducts that can interact efficiently with electron acceptor groups in the polymer, partly responsible for the photochromic degradation. The phthalocyanine molecules seem to protect efficiently the photochromic molecules only at low laser fluences. Moreover, in the case of ultrashort pulses the degradation of the photochromic molecules is found to be more intense that in the case of ns pulses, indicating multiphoton absorption mechanisms, unless low laser fluences are used.
\end{abstract}

(c) 2006 Elsevier B.V. All rights reserved.

Keywords: Polymers; Photochromic molecules; Photodegradation

\section{Introduction}

Absorption of UV light by the photochromic spiropyran (SP) molecules leads to a ring-opening of the molecules, involving cleavage of the $\mathrm{C}-\mathrm{O}$ bond that connects their chromene and indoline parts, and results in the formation of merocyanine (MC) species, which have a strong absorption band in the visible region, due to a larger conjugated $\pi$-electron system (Fig. 1). The formed MC isomers can be transformed back to the SP form either thermally or photochemically [1-4]. These unique properties of the SP photochromic molecules have been widely exploited in a range of applications such as reversible holographic recording [5-7], light-induced waveguides [8], threedimensional optical data storage [9], microfluidics [10], and fast responsive systems, such as microswitches, operating in a

\footnotetext{
* Corresponding author. Tel.: +30 2810391382.

E-mail address: nassia@iesl.forth.gr (A. Athanassiou).
}

fully controllable manner under laser irradiation $[11,12]$. The major restriction factor regarding the above applications of the SP molecules is the photodegradation, which occurs upon irradiation, together with the photochromic processes.

It is widely accepted in the literature that the degradation process in solutions or in solid-state matrices is led mainly by photo-oxidation. In particular, the investigation of the process, in terms of the mechanism responsible, in solution [13-16] and in polymeric matrices $[17,18]$, lead to a number of mechanisms proposed for the oxidative degradation processes, which occur through free radical or singlet oxygen formation [16-18]. In some cases, it was shown that the coexistence of a singlet oxygen quencher, 1,4-diazabicyclo-[2,2,2]-octane (DABCO) [19], or the mixture with an antioxidant [20] exhibits an increase of the fatigue resistance of the photochromic compounds. Moreover, the use of oxygen barrier layer (carboxymethylcellulose) above a naphthopyran or naphthoxazine doped polymer was shown to increase the photofatigue resistance time by a factor of $\sim 1.5-2.0$ [17]. Photodegradation studies also exist that do not connect the 
SP
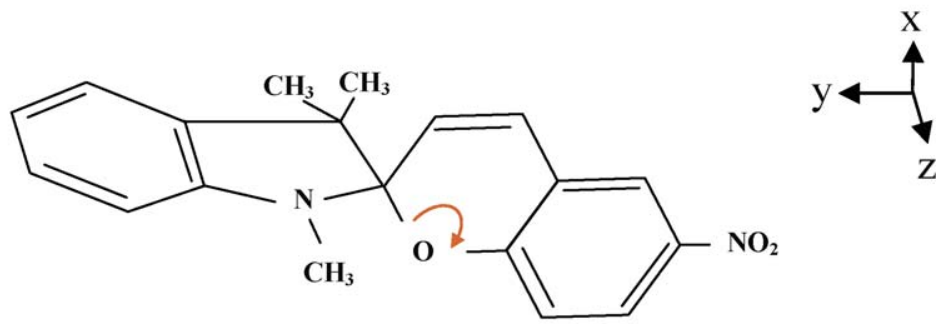

UV

$\uparrow$ 个 Visible, $k T, U V$<smiles>C[N+]1=C(C=Cc2cc([N+](=O)[O-])ccc2[O-])C(C)(C)c2ccccc21</smiles>

TTT<smiles>C[N+]1C(=C=Cc2cc([N+](=O)[O-])ccc2[O-])C(C)(C)c2ccccc21</smiles>

TTC

\section{Trans MC}<smiles>C[N+]1=C(C=Cc2cc([N+](=O)[O-])ccc2[O-])C(C)(C)c2ccccc21</smiles>

CTT<smiles>C[N+]1=C(C=Cc2cc([N+](=O)[O-])ccc2[O-])C(C)(C)c2ccccc21</smiles>

CTC

Fig. 1. Chemical structures and isomerisation reaction between the photochromic SP molecule and its stable isomeric forms of MC.

photodegradation with the presence of oxygen, when the irradiation of the samples occurs at long UV wavelengths close to the visible. For example experiments performed on spirooxanine in toluene with continuous monochromatic irradiation at $365 \mathrm{~nm}$, showed that the degradation occurs possibly from the thermal decomposition of the open merocyanine form, whereas when the same experiments carried out at $313 \mathrm{~nm}$, oxygen was accelerating the photodegradation [21]. Matsushima et al. [22] proved that spin coated PMMA-spiropyran films irradiated with UV at $365 \mathrm{~nm}$ and visible at $436-545 \mathrm{~nm}$ exhibit degradation via aggregation reactions of the coloured forms and not via oxidation reactions.

In this work are examined the possible photodegradation processes using laser pulses of short UV wavelengths (248 and $308 \mathrm{~nm}$ ) on samples consisting of SP molecules in polymer matrices. Since the SP molecules exhibit a wide UV absorption band (Fig. 2(a)), irradiation of the samples at these wavelengths induce the photocoloration process $\mathrm{SP} \rightarrow \mathrm{MC}$, together with, or prior to, the photodegradation processes. Moreover, a comparison on the degradation of the photochromic molecules is presented using different laser pulse duration ( $30 \mathrm{~ns}$ and $500 \mathrm{fs}$ ). The fatigue of the samples is demonstrated to be much more affected by the change in the pulse duration than from the wavelength change. The stability of the samples is improved when pulses of longer durations are used, since possible multipho- ton excitation processes, that can be responsible for new paths leading to photodissociation, are reduced.

In our experiments, the role of the oxidative degradation is studied by examining the efficiency of Iron(II) phthalocya-

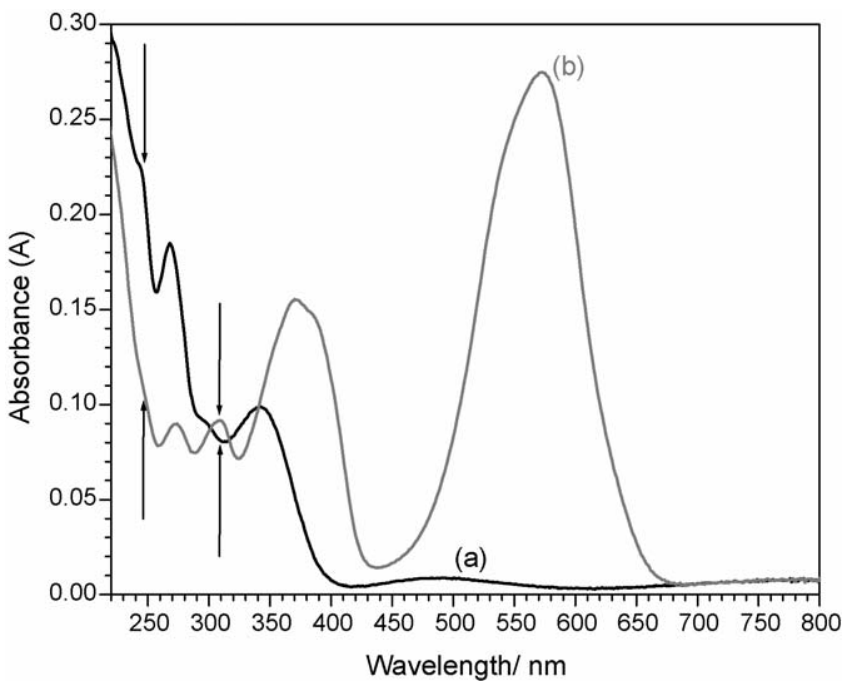

Fig. 2. Absorption spectrum of a film consisting of 5\% SP and 95\% P(EMA)co-P(MA). (a) Before any irradiation; (b) after irradiation with $10 \mathrm{UV}$ pulses of $40 \mathrm{~mJ} \mathrm{~cm}^{-2}$ at $308 \mathrm{~nm}$ with $30 \mathrm{~ns}$ pulse duration. 
nine molecules to maintain the photochromism phenomenon. The idea is that these added molecules can react, instead of the photochromic molecules, with electron acceptor groups in the polymer matrix. Such electron acceptor groups can be found as impurities or radicals in the polymer matrices especially after UV irradiation [23], and can be the cause of the oxidation and degradation of the doped MC molecules. In general, metal ions embodied in protein matrices, such as the Iron(II) ion in haemoglobin, serve numerous important functions in the body related to the metabolism and transport of oxygen. Iron in this process can exist in two oxidation states: ferrous $\left(\mathrm{Fe}^{2+}\right)$ or ferric $\left(\mathrm{Fe}^{3+}\right)$, and exhibits a strong affinity for electronegative atoms as oxygen, nitrogen, and sulfur, which are found at the heart of the iron-binding centres of the macromolecules $[24,25]$. Iron is associated with proteins either by incorporation into protoporphyrin IX or by binding to other ligands. The phthalocyanine molecule, which is used in this work, plays a relevant role in material sciences among tetrapyrrolic macrocycles as synthetic analogue of the porphyrin family [26]. In this work, apart from taking advantage of the strong affinity of the iron binding centre of the phthalocyanine molecule for electronegative groups, we use the Fe(II) phthalocyanine molecules for an additional reason. These molecules are unstable under UV irradiation, and their photochemical decay can include reaction with oxygen molecules or with other groups which lead to the oxidation of the phthalocyanine photoproducts [27]. Therefore, in a indirect way the decay products of the phthalocyanine macrocycle are expected to inhibit the oxidative degradation of the photochromic molecules, since they can react with the deleterious polymeric radicals, instead of the MC molecules.

\section{Experimental description}

The sample under investigation were polymer films doped with photochromic SP molecules with or without the addition of $\mathrm{Fe}$ (II) phthalocyanine molecules. In particular the films used were: (1) $5 \%$ by wt. of the SP molecules in $95 \%$ by wt. of the polymer poly(ethyl methacrylate)-co-poly(methyl acrylate) $\mathrm{P}(\mathrm{EMA})-\mathrm{co}-\mathrm{P}(\mathrm{MA})$, and (2) 5\% by wt. of the SP molecules in $95 \%$ by wt. of a mixture of $\mathrm{Fe}(\mathrm{II})$ phthalocyanine molecules with $\mathrm{P}(\mathrm{EMA})$-co-P(MA). The mixture of the polymer with the phthalocyanine molecules was prepared as follows: few milligrams of phthalocyanine molecules were dissolved during heating and then filtered into the polymer. For the preparation of the films, the above-described solutions of the polymer and of the molecules were prepared in toluene. Certain volume of this solution was spin-coated on quartz substrates, to produce homogeneous films. The achieved thicknesses of the films were in the range of $1.0-3.5 \mu \mathrm{m}$.

The degradation studies were performed by absorption measurements on the prepared films. Initially the absorption spectrum of each film was recorded in a UV-visible scanning spectrophotometer. Next, the film was irradiated with UV laser pulses and after 5-10 successive pulses the absorption spectrum was recorded again. After each absorption measurement, the film was carefully replaced, for the subsequent irradiation, at the previous position, to ensure that the same point on the sample is always irradiated. The overall number of UV pulses used on each sample was 200. The irradiated area was $0.07 \mathrm{~cm}^{2}$. For the irradiation of the samples three different UV lasers were used. The first was a $\mathrm{XeCl}$ laser operating at $308 \mathrm{~nm}$ with $30 \mathrm{~ns}$ pulse duration (Lambda Physik, EMG 201 MSC), the second was a $\mathrm{KrF}$ laser operating at $248 \mathrm{~nm}$ with $30 \mathrm{~ns}$ pulse duration (Lambda Physik, EMG150), and the third was a distributed feedback dye laser system operating at the $\mathrm{KrF}$ excimer wavelength of $248 \mathrm{~nm}$ with 500 fs pulse duration. Three different energy densities were used for the irradiation of the samples with each laser. For the two lasers emitting at $248 \mathrm{~nm}$ the fluences used were 25,15 , and $9 \mathrm{~mJ} \mathrm{~cm}^{-2}$, whereas for the laser emitting at $308 \mathrm{~nm}$ the fluences used were 40,25 , and $15 \mathrm{~mJ} \mathrm{~cm}^{-2}$. The repetition rate of the lasers was $1 \mathrm{~Hz}$ in all cases. The absorption changes to the examined samples upon addition of the $\mathrm{Fe}$ (II) phthalocyanine molecules are considered negligible (see Figure $\mathrm{A}$ in the supporting material), and therefore we assume no interference of the $\mathrm{Fe}(\mathrm{II})$ phthalocyanine molecules in the interconversion processes of the photochromic molecules.

\section{Results}

The photodegradation of the photochromic molecules used in this study is examined exclusively upon UV irradiation. Even after the very first UV laser pulse the studied photochromic films exhibit new absorption peaks which are attributed to the formation of MC stereoisomers. In Fig. 2 is shown the absorption spectrum of a 5\% SP-95\% P(EMA)-co-P(MA) film before and after UV irradiation. The most pronounced absorption peak of the MC molecules is formed in the visible with a maximum around $573 \mathrm{~nm}$. The maximum intensity of the MC absorbance is achieved after a certain number of UV pulses. This is due to the fact that the quantum yield of the $\mathrm{SP} \rightarrow \mathrm{MC}$ conversion, or photocoloration, is less than 1. Indeed, the photocoloration quantum yield in solvents of low polarity is quite high e.g. 0.6 in toluene and 0.8 in cyclohexane [28,29], and therefore we expect a similar, possibly decreased, quantum yield in our system where the SP molecules are hosted in a non-polar but highly viscous medium. In the range of the energy densities used in the presented experiments the maximum absorbance intensity of the peaks attributed to the MC molecules was obtained within 10-20 UV pulses, indicating that the SP to MC coloration process is completed. Graph 1(b) in Fig. 2 illustrates the absorption spectrum when the principal absorption peak of the MC molecules $(573 \mathrm{~nm})$ has reached its maximum intensity.

The formed MC molecules also exhibit absorption in the UV region as demonstrated in graph 1(b). Therefore, after the achievement of the maximum MC absorbance, subsequent UV laser pulses are absorbed by the formed MC molecules. Upon UV absorption these molecules can either undergo isomerisation to other MC stereoisomers, since they can exist in four different stable stereoisomers, which exhibit trans configuration at the bridge segment [1] (see Fig. 1), or they can return to the initial SP isomer. The MC molecules that return to the SP form, upon subsequent UV irradiation will undergo again the coloration transformation to the MC forms. The different trans $\mathrm{MC}$ stereoisomers that can co-exist during this procedure 

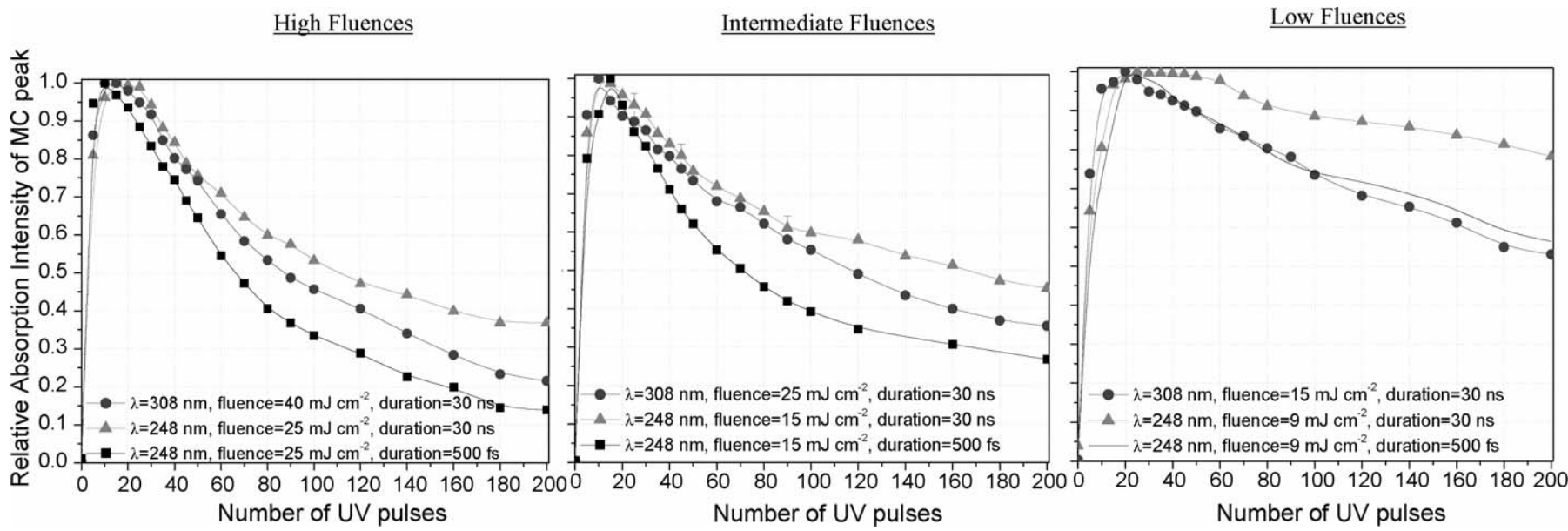

Fig. 3. Relative absorbance of the MC peak at $\sim 573 \mathrm{~nm}$ vs. number of UV pulses for a $5 \%$ by wt. SP in P(EMA)-co-P(MA) film. (a) Comparison between the effect of laser pulses at $(\bullet) \lambda=308 \mathrm{~nm}$, fluence $\sim 40 \mathrm{~mJ} \mathrm{~cm}^{-2}$ and duration $30 \mathrm{~ns} ;(\boldsymbol{\Lambda}) \lambda=248 \mathrm{~nm}$, fluence $\sim 25 \mathrm{~mJ} \mathrm{~cm}^{-2}$ and duration $30 \mathrm{~ns}$; ( $\left.\mathbf{\square}\right) \lambda=248 \mathrm{~nm}$, fluence $\sim 25 \mathrm{~mJ} \mathrm{~cm}^{-2}$ and duration $500 \mathrm{fs}$. (b) Comparison between the effect of laser pulses at () $\lambda=308 \mathrm{~nm}$, fluence $\sim 25 \mathrm{~mJ} \mathrm{~cm}^{-2}$ and duration $30 \mathrm{~ns} ;(\boldsymbol{\Lambda}) \lambda=248 \mathrm{~nm}$, fluence $\sim 15 \mathrm{~mJ} \mathrm{~cm}^{-2}$ and duration $30 \mathrm{~ns}$; (ם) $\lambda=248 \mathrm{~nm}$, fluence $\sim 15 \mathrm{~mJ} \mathrm{~cm}^{-2}$ and duration $500 \mathrm{fs}$. (c) Comparison between the effect of laser pulses at (-) $\lambda=308 \mathrm{~nm}$, fluence $\sim 15 \mathrm{~mJ} \mathrm{~cm}^{-2}$ and duration $30 \mathrm{~ns} ;(\boldsymbol{\Lambda}) \lambda=248 \mathrm{~nm}$, fluence $\sim 9 \mathrm{~mJ} \mathrm{~cm}^{-2}$ and duration $30 \mathrm{~ns} ;(\boldsymbol{\square}) \lambda=248 \mathrm{~nm}$ with fluence $\sim 9 \mathrm{~mJ} \mathrm{~cm}{ }^{-2}$ and duration $500 \mathrm{fs}$.

exhibit similar spectroscopic behaviour, and thus, they cannot be distinguished by their absorption spectra. Therefore, in an ideal system, after the initial number of pulses needed for the completion of the coloration process, the following UV pulses should keep the SP-MC population in equilibrium, and so, the intensity of the MC absorption peak should remain unaltered. Instead, the intensity of this peak decreases upon continuous irradiation with UV pulses, and this decrease is due to the photodegradation of the MC molecules (Fig. 3). The thermal reversion of the MC forms to the SP under the experiment conditions happens in few days, significantly longer time than this of the measurements, and therefore any decrease of the MC absorption peak is attributed exclusively to photodegradation phenomena.

For the study of the photodegradation resistance of the samples, two UV wavelengths $(248,308 \mathrm{~nm})$ were used. In these wavelengths the samples exhibit different absorbance values, as clearly shown in Fig. 2. The absorbed light intensity in the samples is given by Beer's law:

$\frac{I_{\mathrm{t}}}{I_{\mathrm{i}}}=\mathrm{e}^{-2.303 A} \Rightarrow \frac{I_{\mathrm{i}}-I_{\mathrm{a}}}{I_{\mathrm{i}}}=\mathrm{e}^{-2.303 A} \Rightarrow I_{\mathrm{a}}=I_{\mathrm{i}}\left(1-\mathrm{e}^{-2.303 A}\right)$

where $I_{\mathrm{i}}$ is the incident light intensity, $I_{\mathrm{t}}$ the transmitted light intensity, $I_{\mathrm{a}}$ the absorbed light intensity in the sample, and $A$ is the absorbance of the sample which depends on the wavelength.

For comparison reasons of the degradation of the photochromic molecules at the two different wavelengths, the incident light intensity in the two examined cases is calculated in such a way that the absorbed light intensity in the samples is equal:

$I_{\mathrm{a}(248 \mathrm{~nm})}=I_{\mathrm{a}(308 \mathrm{~nm})}$

Therefore, the relation between the incident light intensities at the two examined wavelengths is calculated by the Eq. (3) extracted by Eqs. (1) and (2)

$\frac{I_{\mathrm{i}(308 \mathrm{~nm})}}{I_{\mathrm{i}(248 \mathrm{~nm})}}=\frac{1-\mathrm{e}^{-2.303 A_{248 \mathrm{~nm}}}}{1-\mathrm{e}^{-2.303 A_{308 \mathrm{~nm}}}}$

There is a dynamic change in the absorbance $(A)$ of the samples upon UV irradiation since the newly formed MC molecules after each pulse absorb differently than the initial SP molecules. Therefore, we calculated the intensity ratios from Eq. (3), throughout the irradiation process (with laser pulses of $40 \mathrm{~mJ} \mathrm{~cm}^{-2}$ at $308 \mathrm{~nm}$ ), starting from the initial sample and finishing with the sample after the completion of the SP to MC coloration process. Finally, the intensities used in the experiments were determined by the average value of the calculated ratios, which is: $I_{\mathrm{i}(308 \mathrm{~nm})} \sim 1.66 \times I_{\mathrm{i}(248 \mathrm{~nm})}$. It can be considered as a rough calculation for equally absorbed light intensities in the samples using either 248 or $308 \mathrm{~nm}$ laser pulses. The comparison of the degradation of the photochromic molecules caused by laser pulses of either 248 or $308 \mathrm{~nm}$ at fluences determined by the afore-calculated relationship is demonstrated in Fig. 3.

The photodegradation resistance of the SP doped polymer films is described by the changes in the relative absorption intensity $A_{n} / A_{\max }$ upon irradiation with UV pulses (Fig. 3), where, $A_{n}$ represents the absorbance value of the MC forms in the photostationary state obtained after the $n$th UV laser pulse, and $A_{\max }$ represents the maximum MC absorbance value (usually obtained after the 10th-20th pulse for the used fluences). A comparison, between the different fluence ranges used, clearly indicates that decrease of the laser fluence results into enhanced resistance of the photochromic molecules to the photodegradation. Furthermore, the degradation effect appears quite enhanced in the case of the $308 \mathrm{~nm}$ pulses compared to the $248 \mathrm{~nm}$ especially at low laser fluences, after the normalization of the incident laser intensities for equally absorbing samples at the two wavelengths. Finally, Fig. 3 demonstrates that the fs pulses induce a higher degree of fatigue in the samples, which is even more 
evident at high laser fluences. In particular, comparing laser pulses of $248 \mathrm{~nm}$ wavelength and different pulse durations, it is obvious that the ultrashort pulses are always responsible for enhanced degradation compared to the pulses of longer pulse duration.

The above-presented samples with added phthalocyanine molecules in the matrix were also examined under irradiation with different laser conditions. The phthalocyanine molecules and especially their UV photolysis products can react, instead of the MC molecules, with electron acceptor groups in the polymer, preventing the oxidative degradation of the photochromic molecules. It was found that the addition of the phthalocyanine molecules improves the degradation resistance of the system only under specific irradiation conditions, as shown in Fig. 4. The photodegradation is again described by the changes in the relative absorption intensity $A_{n} / A_{\max }$ upon irradiation with $\mathrm{UV}$ pulses, and for comparison reasons in the figure are presented the results for samples with and without $\mathrm{Fe}(\mathrm{II})$ phthalocyanine molecules. In particular in Fig. 4(a) is demonstrated that the added molecules are unable to prevent the degradation of the photochromic molecules, at high fluences. On the contrary in Fig. 4(b) and (c) it is demonstrated that the resistance of the system is improved going towards lower fluences for the laser pulses of $30 \mathrm{~ns}$ duration. In the case of irradiation with $248 \mathrm{~nm}$ pulses of $500 \mathrm{fs}$ duration, the system is only improved using the $\mathrm{Fe}(\mathrm{II})$ phthalocyanine molecules at very low fluences.

\section{Discussion}

For thorough discussion and comparison of the results, they are summarized in Fig. 5. In this figure is plotted the photodegradation of the samples with (full symbols) and without $\mathrm{Fe}$ (II) phthalocyanine molecules (empty symbols) for the different ranges of laser fluences used. The photodegradation is represented in percentage reduction of the maximum MC absorbance. The values are calculated using the following formula $\left(\frac{A_{\max }-A_{n}}{A_{\max }}\right) \times 100 \%$, where as $n$ we always use 160 pulses. There is clear evidence that the photodegradation depends on the different irradiation wavelengths and the different pulse durations.

At the two different wavelengths used (248 and $308 \mathrm{~nm}$ ), the degradation effect appears quite enhanced in the case of the $308 \mathrm{~nm}$ pulses compared to the $248 \mathrm{~nm}$ especially at low fluences. In this fluence regime, the MC absorbance after irradiation with $160 \mathrm{UV}$ pulses of $248 \mathrm{~nm}$ ( $30 \mathrm{~ns}$ pulse duration) is $\sim 16 \%$ reduced compared to the maximum absorbance, whereas in the case of $308 \mathrm{~nm}$ pulses the reduction is much higher, $\sim 35 \%$ (Figs. 3 and 5).

For the two wavelengths the incident intensities were normalized, as described above, for equally absorbing samples. It should be commented here that the polymer used is a stronger absorber at $248 \mathrm{~nm}$ than at $308 \mathrm{~nm}$. The difference in the absorption at the two wavelengths is profoundly enhanced in the case of the pure polymer (Fig. 6) compared to the polymer doped with photochromic molecules (Fig. 2). For the doped polymer, the difference is continuously decreasing as the MC molecules are formed. Therefore, although the samples absorb equal number of photons at the two wavelengths, the polymer absorbs more photons at $248 \mathrm{~nm}$, and eventually the photochromic molecules absorb more photons at $308 \mathrm{~nm}$. The increased absorption at $308 \mathrm{~nm}$ of the photochromic molecules leads in the faster formation of the different photostationary states of the MC molecules, and consecutively in their faster degradation rate, since it is well acknowledged in the literature that the degradation of the photochromic system occurs through the open MC form via different possible mechanisms (oxidation, thermal decomposition, aggregation $[17,18,21,22])$.

A further plausible reason for the different degradation rate of the photochromic molecules upon irradiation with laser pulses at 248 and $308 \mathrm{~nm}$ might be connected to non-linear mechanisms. Multiphoton processes are highly possible, even in the case of ns pulse durations, since triplet excited states are involved in the photochromic transformation of the examined molecule. In particular, the excited states were found to last up to few tens of ns in the case of the first triplet states [28,29,30], and $57 \mathrm{ps}$ in the case of the $\mathrm{MC} \mathrm{S}_{1}$ state [31]. Furthermore, it is reported time up to $0.2 \mathrm{ps}$ for the absorption from the first excited singlet state to higher excited singlet states $\left(S_{1} \rightarrow S_{n}\right)$ of the SP molecules [31]. Multiphoton absorption can lead to degradation photoproducts directly formed in the high-energy states of the photochromic molecules, either by photodissociation or making the oxidation pathways more efficient. In our system is expected that more photochromic molecules undergo the non-linear excitation upon irradiation with $308 \mathrm{~nm}$ than with $248 \mathrm{~nm}$ pulses, since more photons are absorbed by the molecules at $308 \mathrm{~nm}$. Thus more molecules undergo degradation due to non-linear excitation after $308 \mathrm{~nm}$ irradiation, because part of the incident photons at $248 \mathrm{~nm}$ are absorbed by the polymer, which does not undergo any multiphoton absorption at this pulse duration at least in the range of the fluences used [32]. It is expected that reducing the fluence, the protective role of the polymer upon $248 \mathrm{~nm}$ irradiation becomes even more evident since the remaining number of photons absorbed by the photochromic molecules becomes insufficient to induce excitation to higher excited sates.

A final argument that can support higher degradation rates in the case of irradiation with $308 \mathrm{~nm}$ can be found in the work by Torikai et al. [33]. They use different UV wavelengths for the irradiation of an acrylate polymer, the PMMA, and they found that for irradiation at $300 \mathrm{~nm}$ the quantum yield for chain scission and radical formation was doubled compared to irradiation at $260 \mathrm{~nm}$. Therefore, it is plausible an enhanced radical formation in the case of $308 \mathrm{~nm}$ irradiation which lead to oxidative degradation of the MC molecules, since the polymeric radicals can be strong electron acceptor groups which oxidize the MC molecules.

Following the above discussed argument of multiple-step absorption of photons during the laser pulses which leads to enhanced photodegradation of the system, these processes are more efficient in the case of $500 \mathrm{fs}$ pulse duration and this explains the enhanced degradation of the system upon irradiation with fs pulses. The protective role of the polymer, which decreases abruptly the degradation of the system at low fluences 

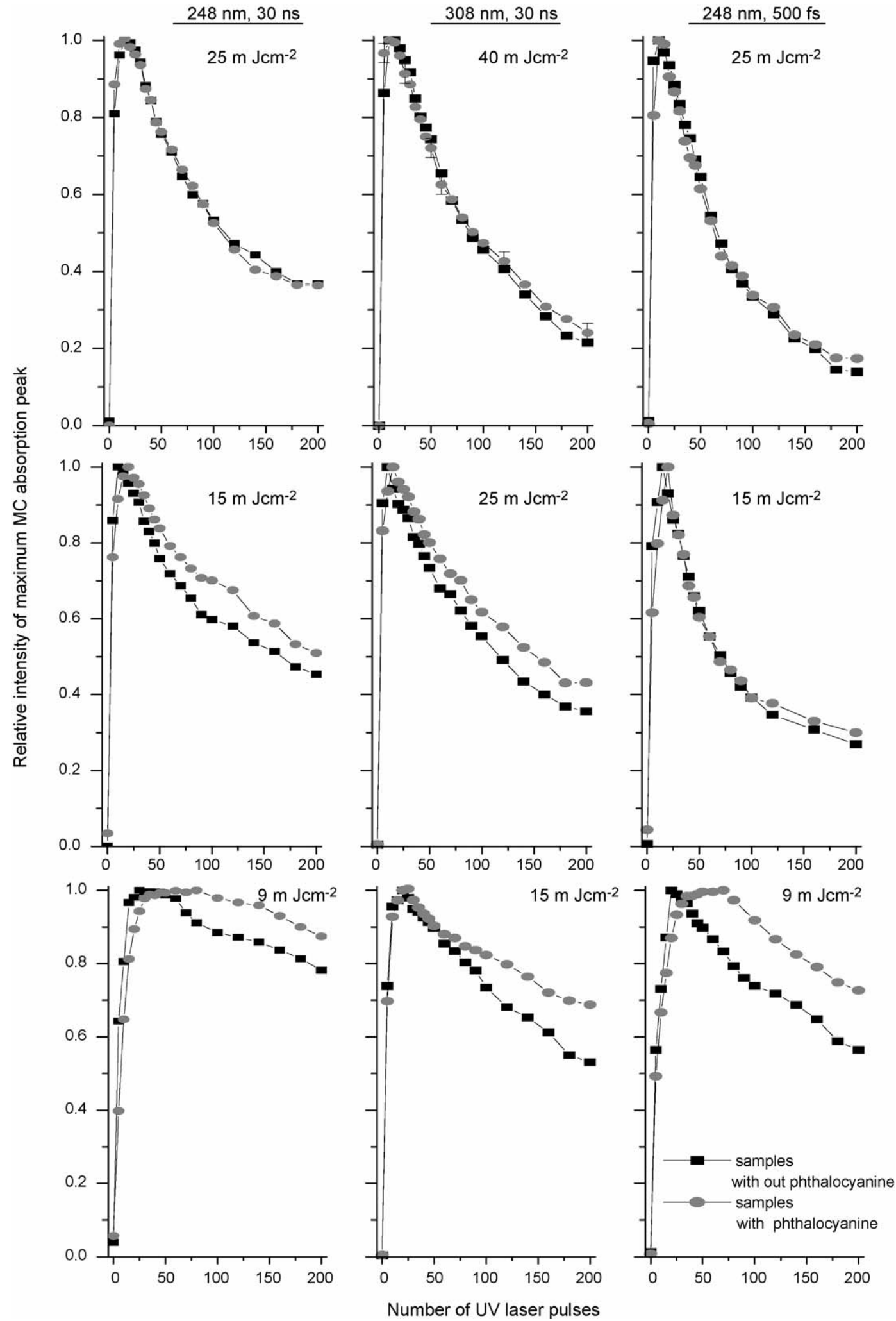

Fig. 4. Comparison of the relative absorbance of the MC peak at $\sim 573 \mathrm{~nm}$ vs. number of UV pulses between a $5 \%$ by wt. SP in P(EMA)-co-P(MA) film ( $\square$ ) and the same film with added phthalocyanine molecules (๑). (a) High fluence regime $\sim 40 \mathrm{~mJ} \mathrm{~cm}^{-2}$ at $\lambda=308 \mathrm{~nm}$ and $\sim 25 \mathrm{~mJ} \mathrm{~cm}^{-2}$ at $\lambda=248 \mathrm{~nm}$. (b) Intermediate fluence regime $\sim 25 \mathrm{~mJ} \mathrm{~cm}^{-2}$ at $\lambda=308 \mathrm{~nm}$ and $\sim 15 \mathrm{~mJ} \mathrm{~cm}^{-2}$ at $\lambda=248 \mathrm{~nm}$. (c) Low fluence regime $\sim 15 \mathrm{~mJ} \mathrm{~cm}^{-2}$ at $\lambda=308 \mathrm{~nm}$ and $\sim 9 \mathrm{~mJ} \mathrm{~cm}^{-2}$ at $\lambda=248 \mathrm{~nm}$. 


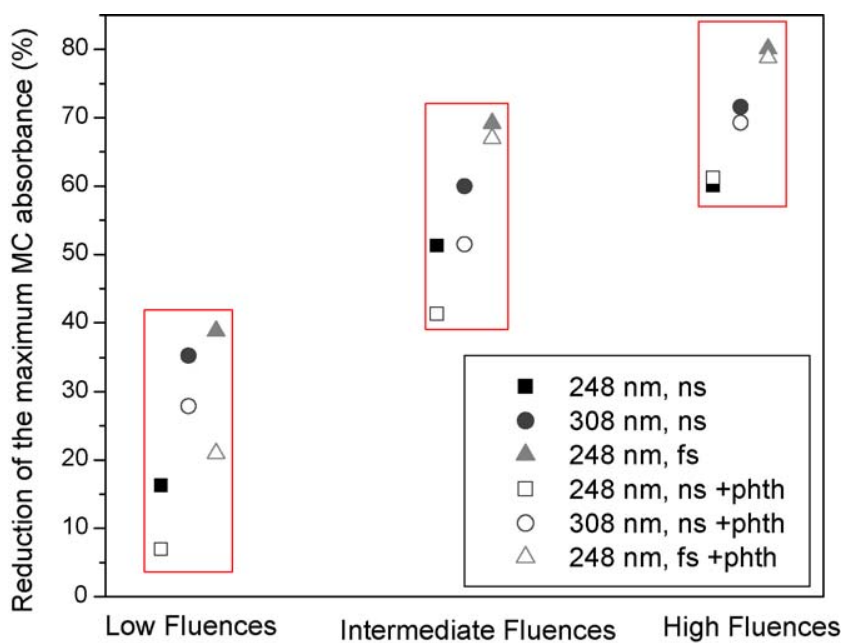

Fig. 5. Percentage reduction of the maximum MC absorbance after irradiation with 160 UV laser pulses, of samples 5\% by wt. SP in P(EMA)-co-P(MA) with (full symbols) and without (empty symbols) Fe(II) phthalocyanine molecules, for the different ranges of laser fluences used. High fluences: $40 \mathrm{~mJ} \mathrm{~cm}^{-2}$ at $\lambda=308 \mathrm{~nm}$ and $25 \mathrm{~mJ} \mathrm{~cm}^{-2}$ at $\lambda=248 \mathrm{~nm}$. Intermediate fluences: $25 \mathrm{~mJ} \mathrm{~cm}^{-2}$ at $\lambda=308 \mathrm{~nm}$ and $15 \mathrm{~mJ} \mathrm{~cm}^{-2}$ at $\lambda=248 \mathrm{~nm}$. Low fluences: $15 \mathrm{~mJ} \mathrm{~cm}^{-2}$ at $\lambda=308 \mathrm{~nm}$ and $9 \mathrm{~mJ} \mathrm{~cm}^{-2}$ at $\lambda=248 \mathrm{~nm}$.

upon $248 \mathrm{~nm}$ compared to $308 \mathrm{~nm}$ irradiation, is valid even for the short pulse durations of $500 \mathrm{fs}$.

Some additional information elucidating the degradation processes upon UV laser irradiation can be obtained by the photodegradation rates of the photochromic molecules after the addition of the $\mathrm{Fe}$ (II) phthalocyanine to the systems, which are also summarized in Fig. 5. It is plausible that the Fe(II) phthalocyanine molecules coordinate oxygen in correspondence of the iron centre when exposed on oxygen-containing atmosphere, and therefore possibly in some degree they contain oxygen before being embedded in the polymeric film [34]. According to Slota and Dydra [27] the oxygen-containing or oxygen-free phthalocyanine molecules remain stable unless exposed to UV light. Due to absorption of UV radiation the presumable Fe(II)

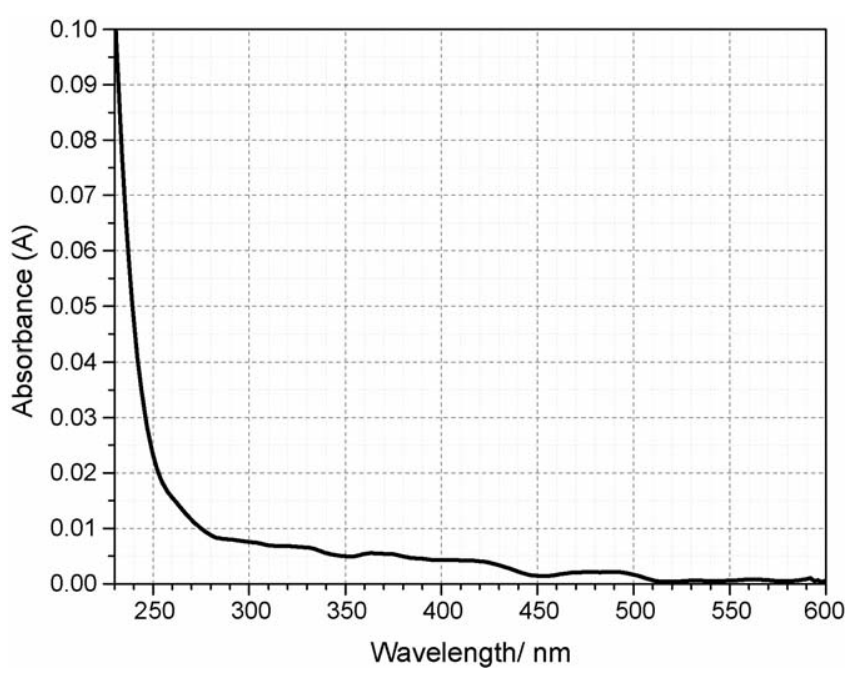

Fig. 6. Absorption spectrum of the polymer P(EMA)-co-P(MA). It exhibits an enhanced absorption at $248 \mathrm{~nm}$ compared to $308 \mathrm{~nm}$. phthalocyanine- $\mathrm{O}_{2}$ bond is supposed to give off the oxygen molecule and the phthalocyanine molecule to photodissociate. The decay photoproducts of the phthalocyanine are possible to react either with the oxygen molecules, released or already present in the polymer matrix, or with electron acceptor groups present in the matrix by interactions of the phthalocyanine macrocycle bridging nitrogen atoms [27]. These reactions of the phthalocyanine molecules are expected to protect indirectly the photochromic molecules from the oxidative degradation.

As demonstrated in Figs. 4 and 5, for high fluences it is clear that the degradation resistance does not change after the addition of the $\mathrm{Fe}$ (II) phthalocyanine, indicating that the oxidative degradation of the system is not prevented due to enhanced number of electron acceptor groups formed in the polymer at these fluences, or due to direct molecules dissociation from the highly excited states reached after non-linear absorption. At intermediate laser fluences it seems that the oxidative photodegradation, after linear excitation of the molecules, plays an important role for irradiation with laser pulses of $30 \mathrm{~ns}$ duration, since the $\mathrm{Fe}$ (II) phthalocyanine photoproducts start to become effective in the protection of the system. At this range of fluences the multiphoton excitations, and thus the alternative pathways of dissociation of the photochromic molecules, are still very efficient for the pulses of 500 fs duration and therefore, the Fe(II) phthalocyanine photoproducts are not capable to protect the system from degradation. Finally, at low fluences, the Fe(II) phthalocyanine molecules improve the degradation resistance of the samples even in the case of the fs laser pulses indicating limitation of the multiphoton processes and simultaneous occurrence of oxidative photodegradation. In the case of ns laser pulses of $248 \mathrm{~nm}$, the MC absorbance after irradiation with $160 \mathrm{UV}$ pulses is less that $10 \%$ reduced compared to the maximum absorbance, indicating an important enhancement in the degradation resistance of the system.

\section{Conclusions}

Photodegradation studies were performed by means of absorption measurements on polymer films doped with photochromic 6-nitrospiropyran molecules under irradiation with ultraviolet (UV) laser pulses of wavelength 248 and $308 \mathrm{~nm}$, and pulse duration $30 \mathrm{~ns}$ and $500 \mathrm{fs}$ in the first case, and $30 \mathrm{~ns}$ in the second case. Incorporation of Iron(II) phthalocyanine molecules in the examined matrices was used to tackle the problem of oxidative photodegradation. Irradiation at $308 \mathrm{~nm}$ leads to faster degradation rate of the photochromic molecules compared to $248 \mathrm{~nm}$ irradiation, mainly due to the increased absorption of the molecules at $308 \mathrm{~nm}$ resulting in the faster formation of the different photostationary states of the $\mathrm{MC}$ molecules which are susceptible to degradation. The photodegradation seems to be also connected with excitation of the photochromic molecules to high excited states when high laser fluences are used and especially in the case of the short pulse durations. The oxidative degradation process, after linear excitation of the molecules, seems to be dominant at low fluences, where the indirect protection of the system through the phthalocyanine photoproducts becomes efficient. 


\section{Appendix A. Supplementary data}

Supplementary data associated with this article can be found, in the online version, at doi:10.1016/j.jphotochem.2006.03.016.

\section{References}

[1] N.P. Ernsting, T. Arthen-Engeland, J. Phys. Chem. 95 (1991) 5502-5509.

[2] J. Hobley, V. Malatesta, R. Millini, L. Montanari, W. O’Neil Parker Jr., Phys. Chem. Chem. Phys. 1 (1999) 3259-3267.

[3] A.K. Chibisov, H. Görner, J. Photochem. Photobiol. A 105 (1997) 261-267.

[4] H. Görner, Phys. Chem. Chem. Phys. 3 (2001) 416-423.

[5] A. Tork, F. Boudreault, M. Roberge, A.M. Ritcey, R.A. Lessard, T.V. Galstian, Appl. Optics 40 (2001) 1180-1186.

[6] V. Weiss, A.A. Friesem, V.A. Krongauz, Opt. Lett. 18 (1993) 1089-1092.

[7] G. Berkovic, V. Krongauz, V. Weiss, Chem. Rev. 100 (2000) 1741-1753.

[8] S. Lecompte, U. Gubler, M. Jäger, Ch. Bosshard, G. Montemezzani, P. Günter, L. Gobbi, F. Diederich, Appl. Phys. Lett. 77 (2000) 921-923.

[9] S. Kawata, Y. Kawata, Chem. Rev. 100 (2000) 1777-1788.

[10] R. Rosario, D. Gust, M. Hayes, F. Jahnke, J. Springer, A. Garcia, Langmuir 18 (2002) 8062-8069.

[11] A. Athanassiou, K. Lakiotaki, M. Kalyva, S. Georgiou, C. Fotakis, Appl. Surf. Sci. 248 (2005) 56-61.

[12] A. Athanassiou, M. Kalyva, K. Lakiotaki, S. Georgiou, C. Fotakis, Adv. Mater. 17 (2005) 988-992.

[13] A. Kellmann, F. Tfibel, R. Dubest, P. Levoir, J. Aubard, E. Pottier, R. Guglielmetti, J. Photochem. Photobiol. A 49 (1989) 63-73.

[14] C. Bohne, M.G. Fan, Z.J. Li, Y.C. Liang, J. Lusztyk, J.C. Scaiano, J. Photochem. Photobiol. A 66 (1992) 79-90.

[15] G. Baillet, G. Giusti, R. Guglielmetti, J. Photochem. Photobiol. A 70 (1993) 157-161.
[16] G. Baillet, M. Campredon, R. Guglielmetti, G. Giusti, C. Aubert, J. Photochem. Photobiol. A 83 (1994) 147-151.

[17] G. Baillet, Mol. Cryst. Liq. Cryst. 298 (1997) 75-82.

[18] G. Baillet, G. Giusti, R. Guglielmetti, Bull. Chem. Soc. Jpn. 68 (1995) $1220-1225$.

[19] C. Salemi, G. Giusti, R. Guglielmetti, J. Photochem. Photobiol. A 86 (1995) 247-252.

[20] X. Li, J. Li, Y. Wang, T. Matsuura, J. Meng, J. Photochem. Photobiol. A 161 (2004) 201-213.

[21] V. Pimienta, C. Froute, M.H. Deniel, D. Lavabre, R. Guglielmetti, J.C. Micheau, J. Photochem. Photobiol. A 122 (1999) 199-204.

[22] R. Matsushima, M. Nishiyama, M. Doi, J. Photochem. Photobiol. A 139 (2001) 63-69.

[23] C. Decker, K. Zahouily, Polym. Degrad. Stabil. 64 (1999) 293-304.

[24] K.M. Smith (Ed.), Porphyrins and Metalloporphyrins, Elsevier, Amsterdam, 1975.

[25] J. Deisenhofer, J.R. Norris (Eds.), The Photosynthetic Reaction Center, Academic Press, San Diego, 1993.

[26] R.E. Blankenship, M.T. Madigan, C.E. Bauer (Eds.), Anoxygenic Photosynthetic Bacteria, Kluwer Academic Publishing, Dordrecht, The Netherlands, 1995.

[27] R. Slota, G. Dydra, Inorg. Chem. 42 (2003) 5743-5750.

[28] A.K. Chibisov, H. Görner, J. Phys. Chem. A 101 (1997) 4305-4312.

[29] H. Görner, Chem. Phys. 222 (1997) 315-329.

[30] C. Lenoble, R.S. Becker, J. Phys. Chem. 90 (1986) 62-65.

[31] A.-K. Holm, O.F. Mohammed, M. Rini, E. Mukhtar, E.T.J. Nibbering, H. Fidder, J. Phys. Chem. A 109 (2005) 8962-8968.

[32] A. Athanassiou, E. Andreou, D. Fragouli, D. Anglos, S. Georgiou, C. Fotakis, J. Photochem. Photobiol. A 145 (2001) 229-236.

[33] A. Torikai, M. Ohno, K. Fueki, J. Appl. Polym. Sci. 41 (1990) 1023-1032.

[34] E. Kuzmann, Z. Homonnay, A. Vertes, S. Li, H. Yin, Y. Wei, A. Nath, X. Chen, J. Li, J. Solid State Chem. 170 (2003) 118-123. 